\title{
D02
}

\section{Design of a Complete Characterisation of Bentonite Behaviour under High Pressure and High Temperature}

\author{
M. Rizzi (Ecole Polytechnique Fédérale de Lausanne (EPFL)), S. Salager \\ (EPFL), P. Marschall (NAGRA) \& L. Laloui* (EPFL)
}

\section{SUMMARY}

Repositories in deep clay geological formations are considered one of the most promising solutions for a sustainable management of High Level radioactive Waste (HLW). The Swiss HLW disposal concept consists in horizontal tunnels excavated at high depth in strongly over consolidated clay (Opalinus Clay) where Granular Bentonite is chosen for enclosing steel canisters containing the waste. This paper presents the theoretical approach and the research activities aiming at investigating the behaviour of this material. An elasto-plastic constitutive model taking into account coupled processes of stress, capillary pressure, and temperature is used.

In this framework, from an experimental point of view, an exhaustive characterization is necessary in order to calibrate required parameters and to validate the model. Laboratory tests designed for this purpose are described.

Particular attention is paid in investigating the swelling behaviour, which is among the features that distinguish materials potentially usable in nuclear waste disposals. First results confirm indeed that the chosen bentonite shows a natural and remarkable swelling attitude. 


\section{Introduction}

The long term safety of deep geological repositories for High Level radioactive Waste (HLW) is based on the existence of a series of superposed natural and artificial barriers, constituted by host rocks, a metallic container filled with the waste and other sealing materials surrounding it. In the specific case of the Swiss HLW disposal concept it is envisioned to create horizontal tunnels at high depth in the Opalinus Clay. The choice of buffer material to enclose the steel canisters falls on MX-80 granular bentonite. The aim of this paper is to present a theoretical framework adapted to the bentonite and to provide an experimental program leading to completely characterize the behaviour of the material under high pressure and high temperature. In the following, after a presentation of the general framework and the introduction of the constitutive model, the planned experimental campaign aiming at obtaining reliable results, necessary to calibrate required parameters and to validate the model, is presented. Some results from swelling tests close the paper by way of example.

\section{Granular bentonite in deep repositories}

Bentonite is a highly plastic clay, mainly composed of the smectite mineral montmorillonite. What makes this material potentially usable in nuclear waste disposals are two distinctive properties: the high ion exchange predisposition (Karnland et al. 2006) and the natural swelling capacity, which may provide sufficient sealing properties, allowing filling the tunnel without gaps and limiting the possible damage by restoring the buffer continuity.

Besides the peculiarity of the material, the fact that it will be submitted to various processes ascribable to different kinds of loadings has to be taken into account. Bentonite will undergo a mechanical loading, related to the geometry of the repository; a thermal loading, associated to the heat transfer from the canister to the environment and depending on the radioactive decay of the nuclear waste; and a hydraulic loading, related to the saturation associated to the groundwater drawn from the host rock and to the concomitant desiccation process near the hot canister. Moreover, the coupling of these aspects has to be taken into account. The analysis of coupling effects between thermal, hydraulic, and mechanical loadings is necessary to properly understand the behaviour of the material.

\section{Theoretical framework: ACMEG-TS constitutive model}

The basis from which the constitutive framework derives, is the family of models developed at the Swiss Federal Institute of Technology Lausanne in the last decade (ACMEG-TS). The model is capable of reproducing the behaviour of soil submitted to coupled processes of temperature, stress and suction loading-unloading and considers, in an elasto-plastic framework, the concept of multiple mechanisms of plasticity. The stress-strain and the retention models are coupled by means of the generalized effective stress formulation and key parameters such as preconsolidation pressure (Nuth et al. 2008). The basic idea is to catch the behaviour of the bentonite, mainly in terms of swelling capability, in a high pressure - high temperature - high suction coupled context.

\section{Experimental campaign}

In addition to the fundamental basic characterisation, a series of tests has been designed in order to extract constitutive data and to assess the temperature and suction effects on the mechanical behaviour of the bentonite.

For this purpose, oedometric tests including unloading-reloading cycles (at two different levels of temperature and suction, in order to investigate their influence on the preconsolidation pressure, and on the compression and swelling indices) have to be run. Besides, an added value is brought by other oedometric tests, aiming at investigating the thermo-plasticity of the material on one hand, and the role of secondary consolidation, which is a non-negligible aspect for such a plastic material, on the other hand.

The experimental campaign must also include triaxial tests under well-controlled temperature/stress/pore pressure conditions. The general objective is to complete the evaluation of elastic and plastic parameters and their possible dependence on suction and confining pressure and to provide information about the critical state (in particular its 


\section{Fault and Top Seals

dependence on temperature and confining pressure) and the non-linearity of the elastic behaviour. The idea is to follow wetting and drying under constant load and loading under constant suction, at different temperatures. Our testing program will be enriched by complex loading paths, taking advantage of two triaxial testing devices able to perform tests on unsaturated materials in a very wide range of suction, temperature and confining stress values. By means of drying-wetting cycles at different temperatures, the hydraulic behaviour of the material can be defined (in terms of air entry value, slope of retention curve and extension of the hysteresis) and the thermo-hydraulic coupling analysed.

Finally, a particular attention is paid to specific tests aiming at exploring the swelling behaviour of the bentonite. Their importance lies in the fact that the swelling capacity should be completely characterized since it is one of the properties which makes bentonite a suitable sealing material. All soils containing clay minerals are indeed characterized by a high deformability on hydration. What distinguishes bentonite from other clay minerals is the amplitude of its swelling behaviour and its sensitivity to test conditions.

\section{A focus on confined swelling tests}

Swelling pressure tests make possible to analyse the wetting process and to determine the pressure applied by the material during complete saturation under isochoric conditions.

The specimen is compacted uniaxially directly in the oedometric cells, the initial dry density being chosen to simulate in situ conditions. Figure 1 shows some stress paths followed during the uniaxial compaction phase. The bottom of the sample contained in the oedometric rings is in contact with a porous stone connected to a graduated burette which allows controlling the pore water pressure while the material saturates. The air in the pores escapes from the upper part of the cell through the opportunely pierced piston.

Performed confined tests took a significant amount of time before reaching equilibrium (about 3 months) and showed a high swelling pressure (for instance, for an initial dry density of 1.65 $\mathrm{g} / \mathrm{cm}^{3}$, a value of $3 \mathrm{MPa}$ has been measured), as presented in Figure 2.

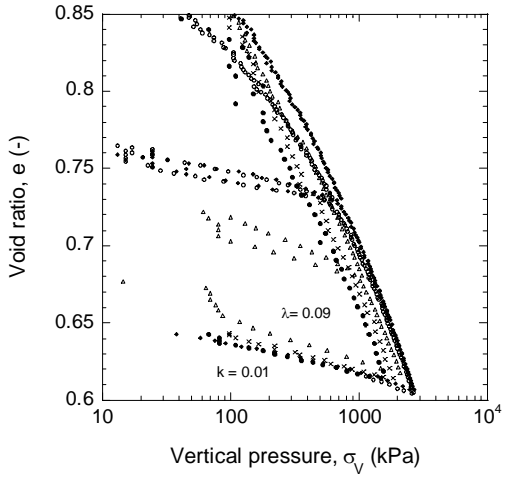

Figure 1- Uniaxial compaction of the samples in oedometric conditions.

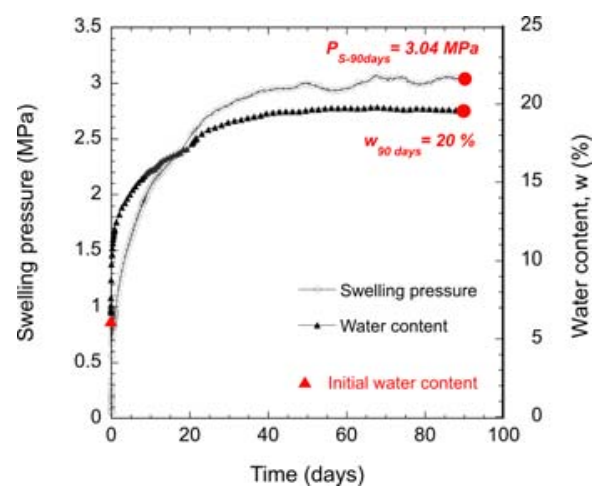

Figure 2- Confined swelling test: Swelling pressure content vs. Time.

\section{References:}

Karnland, O., Olsson, S., and Nilsson, U. [2006] Mineralogy and sealing properties of various bentonites and smectite-rich clay materials. SKB Technical Report, TR, 06-30.

Nuth, M., Laloui, L. [2008] Effective stress concept in unsaturated soils: Clarification and validation of a unified framework. International journal for numerical and analytical methods in Geomechanics, 32, 771-801. 